\title{
THE ALTERNATIVE DUNFORD-PETTIS PROPERTY ON PROJECTIVE TENSOR PRODUCTS
}

\author{
ANTONIO M. PERALTA AND IGNACIO VILLANUEVA
}

\section{INTRODUCTION}

In 1953 A. Grothendieck introduced the property known as DunfordPettis property [18]. A Banach space $X$ has the Dunford-Pettis property (DPP in the sequel) if whenever $\left(x_{n}\right)$ and $\left(\rho_{n}\right)$ are weakly null sequences in $X$ and $X^{*}$, respectively, we have $\rho_{n}\left(x_{n}\right) \rightarrow 0$. It is due to Grothendieck that every $C(K)$-space satisfies the DPP. Historically, were Dunford and Pettis who first proved that $L^{1}(\mu)$ satisfies DPP. Since its introduction, the DPP has been intensively studied and developed in many classes of Banach spaces.

In the last twenty years the problem of determine when the projective tensor product of two Banach spaces satisfies the Dunford-Pettis property has focussed the attention of several researchers.

Since the DPP is inherited by complemented subspaces, it follows that $X$ and $Y$ satisfy the DPP whenever $X \hat{\otimes}_{\pi} Y$ has this property. However, M. Talagrand showed in [26] that this necessary condition is not always sufficient finding a Banach space $X$ such that $X^{*}$ has the Schur property and $X^{*} \hat{\otimes}_{\pi} L^{1}[0,1]$ does not satisfy the DPP. In 1987, R. Ryan proved that $X \hat{\otimes}_{\pi} Y$ satisfy the DPP and does not contain a subspace isomorphic to $\ell_{1}$ if and only if $X$ and $Y$ have both properties.

In [4], the authors show that, for infinite compact Hausdorff spaces $K_{1}$ and $K_{2}$, then the projective tensor product of $C\left(K_{1}\right)$ and $C\left(K_{2}\right)$ has the DPP if and only if $K_{1}$ and $K_{2}$ are scattered, equivalently, $C\left(K_{1}\right)$ and $C\left(K_{2}\right)$ do not contain $\ell_{1}$. This result was generalised to the more general setting of $\mathrm{C}^{*}$-algebras and $\mathrm{JB}^{*}$-triples, by showing that the projective tensor product of two Banach spaces $X$ and $Y$ satisfies the DPP and property $(V)$ of Pelczynzki if and only if $X$ and $Y$ have both properties and do not contain $\ell_{1}$. As a consequence it is shown that the projective tensor product of two infinite dimensional $\mathrm{C}^{*}$-algebras or $\mathrm{JB}^{*}$-triples $E$ and $F$ (see definition below) satisfies the DPP if and only if $E$ and $F$ satisfy the DPP and do not contain $\ell_{1}$.

First author partially supported by I+D MCYT project no. BFM2002-01529, and Junta de Andalucía grant FQM 0199 and second author partially supported by I+D MCYT project no. BFM2001-1284. 
In 1997, W. Freedman introduced an strictly weaker version of the DPP, called the alternative Dunford-Pettis property (see [15]). A Banach space $X$ has the alternative Dunford-Pettis property (DP1 in the sequel) if whenever $x_{n} \rightarrow x$ weakly in $X$, with $\left\|x_{n}\right\|=\|x\|=1$, and $\rho_{n} \rightarrow 0$ weakly in $X^{*}$, we have $\rho_{n}\left(x_{n}\right) \rightarrow 0$. Freedman shown in the same paper that DPP and DP1 are equivalent for von Neumann algebras. L. J. Bunce and the first author of the present note prove in [7] that the same result remains true for general $\mathrm{C}^{*}$-álgebras. In the setting of $\mathrm{JBW}^{*}$-triples, it was shown in [1] that a $\mathrm{JBW}^{*}$-triple $W$ has the DP1 if and only if it satisfies the DPP or the Kadec-Klee property (KKP in the sequel).

Recently, F. Cabello, D. Pérez-García and the second author of the present note prove in [9] that when the projective tensor product of two infinite dimensional $C(K)$ fails the DPP it also fails a weaker property by showing that it contains a complemented copy of $\ell_{2}$.

The main goal of this note is to study the DP1 on projective tensor products of $\mathrm{C}^{*}$-álgebras and $\mathrm{JB}^{*}$-triples. The main difference between DPP and DP1 is that DPP is and isomorphic property (that is, it is preserved by isomorphisms) while the DP1 is an isometric property (preserved by surjective linear isometries and not, in general, by isomorphisms) (see [15, Example 1.6]). In the case of DP1, this will add the difficulty of working with isometric conditions on the projective tensor product of two Banach spaces.

In Section 2, we generalise some results obtained in [9] by replacing the $C(K)$ spaces with more general Banach spaces. More concretely, if $E$ and $F$ are Banach spaces such that $E$ contains $c_{0}$ and $F$ contains a $C(K)$ space containing $\ell_{1}$, then $E \hat{\otimes}_{\pi} F$ contains a complemented copy of $\ell_{2}$. As a consequence, we show that the projective tensor product of two $\mathrm{C}^{*}$-algebras or $\mathrm{JB}^{*}$-triples contains a complemented copy of $\ell_{2}$ whenever it fails the DPP.

In Section 3, we completely describe those $\mathrm{C}^{*}$-algebras whose projective tensor product satisfies the DP1. The main tool to solve this problem is Theorem 3.5 where we prove that if $E$ and $F$ are two Banach spaces such that $E$ contains an isometric copy of $c_{0}$ and $F$ contains and isometric copy of $C[0,1]$, then $E \hat{\otimes}_{\pi} F$ does not have DP1. This result allows us to show that if $E$ and $F$ are $\mathrm{JB}^{*}$-triples such that $E$ is not reflexive and $F$ contains $\ell_{1}$, then $E \hat{\otimes}_{\pi} F$ does not have DP1. As a consequence, Corollary 3.7 shows that the projective tensor product of two infinite dimensional $\mathrm{C}^{*}$-algebras $A$ and $B$ satisfies DP1 if and only if $A$ and $B$ have DP1 and contain no copy of $\ell_{1}$, equivalently, $A \hat{\otimes}_{\pi} B$ satisfies DPP.

Notation: Let $X$ be a Banach space. Through the paper we denote by $X^{*}$ the dual space of $X$. If $Y$ is another Banach space, $L(X, Y)$ (respectively, $K(X, Y)$ ) will stand for the Banach space of all bounded linear operators (respectively, compact linear operators) from $X$ to $Y$. We usually write $L(X)$ 
and $K(X)$ instead of $L(X, X)$ and $K(X, X)$, respectively. The projective tensor product of $X$ and $Y$ will be denoted by $X \hat{\otimes}_{\pi} Y$.

A $J B^{*}$-triple is a complex Banach space $E$ equipped with a continuous triple product

$$
\begin{gathered}
\{., ., .\}: E \otimes E \otimes E \rightarrow E \\
(x, y, z) \mapsto\{x, y, z\}
\end{gathered}
$$

which is bilinear and symmetric in the outer variables and conjugate linear in the middle one and satisfies:

(a) (Jordan Identity)

$$
L(x, y)\{a, b, c\}=\{L(x, y) a, b, c\}-\{a, L(y, x) b, c\}+\{a, b, L(x, y) c\},
$$

for all $x, y, a, b, c \in E$, where $L(x, y): E \rightarrow E$ is the linear mapping given by $L(x, y) z=\{x, y, z\}$;

(b) The map $L(x, x)$ is an hermitian operator with non-negative spectrum for all $x \in E$;

(c) $\|\{x, x, x\}\|=\|x\|^{3}$ for all $x \in E$.

Every $\mathrm{C}^{*}$-algebra is a $\mathrm{JB}^{*}$-triple with respect to

$$
\{x, y, z\}=2^{-1}\left(x y^{*} z+z y^{*} x\right),
$$

every $\mathrm{JB}^{*}$-algebra is a $\mathrm{JB}^{*}$-triple with triple product

$$
\{a, b, c\}=\left(a \circ b^{*}\right) \circ c+\left(c \circ b^{*}\right) \circ a-(a \circ c) \circ b^{*},
$$

and the Banach space $B(H, K)$ of all bounded linear operators between two complex Hilbert spaces $H, K$ is also an example of a $\mathrm{JB}^{*}$-triple with respect to $\{R, S, T\}=2^{-1}\left(R S^{*} T+T S^{*} R\right)$.

A subspace $I$ of a $\mathrm{JB}^{*}$-triple $E$ is called a triple ideal if $\{E, E, I\}+$ $\{E, I, E\} \subset I$. If $I$ satisfies that $\{I, E, I\} \subset I$, then $I$ called an inner ideal of $E$.

A $\mathrm{JBW}^{*}$-triple is a $\mathrm{JB}^{*}$-triple which is also a dual Banach space. The bidual of a $\mathrm{JB}^{*}$-triple $E$ is a $\mathrm{JBW}^{*}$-triple with a triple product extending the one on $E$ (compare [14]).

Every $\mathrm{JB}^{*}$-triple is a Jordan triple. Therefore, given a tripotent $e$ (i.e. $\{e, e, e\}=e)$ in a $\mathrm{JB}^{*}$-triple $E$, there exists a decomposition of $E$ in terms of the eigenvalues of $L(e, e)$ :

$$
E=E_{0}(e) \oplus E_{1}(e) \oplus E_{2}(e)
$$

where $E_{k}(e)=\left\{x \in E: L(e, e) x=\frac{k}{2} x\right\}$ for $k=0,1,2 . E_{k}(e)$ is called the Peirce $k$-space of $e$. The following rules are satisfied:

(1) $\left\{E_{i}(e), E_{j}(e), E_{k}(e)\right\} \subseteq E_{i-j+k}(e)$, where $i, j, k=0,1,2$ and $E_{l}(e)=$ 0 for $l \neq 0,1,2$.

(2) $\left\{E_{0}(e), E_{2}(e), E\right\}=\left\{E_{2}(e), E_{0}(e), E\right\}=0$. 
The projection $P_{k}(e)$ of $E$ onto $E_{k}(e)$ is called the Peirce $k$-projection of $e$. These projections are given by

$$
\begin{aligned}
& P_{2}(e)=Q(e)^{2} \\
& P_{1}(e)=2\left(L(e, e)-Q(e)^{2}\right) \\
& P_{0}(e)=I d_{U}-2 L(e, e)+Q(e)^{2}, .
\end{aligned}
$$

where $Q(e)$ is the conjugate linear operator on $E$ defined by

$$
Q(e)(x):=\{e, x, e\} .
$$

Let $E$ be a $\mathrm{JB}^{*}$-triple. A tripotent $e \in E$ is called minimal if $E_{2}(e)$ coincides with $\mathbb{C} e$. Two tripotents $e, f \in E$ are called orthogonal whenever $L(e, f)=0$ (equivalently, $L(f, e)=0$ ).

\section{Complemented COPIes of $\ell_{2}$ In the Projective tensor Product OF BANACH SPACES}

As we said in the introduction, in this section we extend some of the results in [9] to a wider class of Banach spaces, which in particular includes $\mathrm{C}^{*}$-algebras and non reflexive $\mathrm{JB}^{*}$-triples.

We start recalling some known notions. Given $X$ and $1 \leq p \leq \infty$, we say that a (finite or infinite) sequence $\left(x_{n}\right)_{n} \subset X$ is strongly $p$-summable if $\left(\left\|x_{n}\right\|\right)_{n} \in \ell_{p}$. In that case we define its strong $p$-summing norm by

$$
\left\|\left(x_{n}\right)_{n}\right\|_{p}=\left(\sum_{n}\left\|x_{n}\right\|^{p}\right)^{\frac{1}{p}} .
$$

Analogously we say that $\left(x_{n}\right)_{n}$ is weakly $p$-summable if, for every $x^{*} \in X^{*}$, $\left(\left\langle x^{*}, x_{n}\right\rangle\right)_{n} \in \ell_{p}$. In that case we define the weak p-summing norm of $\left(x_{n}\right)$ by

$$
\left\|\left(x_{n}\right)_{n}\right\|_{p}^{\omega}=\sup \left\{\left(\sum_{n}\left\langle x^{*}, x_{n}\right\rangle^{p}\right)^{\frac{1}{p}}: x^{*} \in B_{X^{*}}\right\} .
$$

We recall that, if $1 \leq p<+\infty$, an operator $T: X \longrightarrow Y$ is called $p$-summing it takes weakly $p$-summable sequences to strong $p$-summable sequences, that is, if there exists a constant $K>0$ such that, for every $p$-weak summing sequence $\left(x_{n}\right)_{n} \subset X$

$$
\left(\sum_{n}\left\|T\left(x_{n}\right)\right\|^{p}\right)^{\frac{1}{p}} \leq K\left\|\left(x_{n}\right)\right\|_{p}^{\omega}
$$

In that case, we define the $p$-summing norm of $T$ by $\pi_{p}(T)=\min \{K$ : $K$ verifies (1)\}.

The following result is proved in [9]. 
Lemma 2.1. Let $\left(b_{n}\right) \subset C\left(K_{2}\right)$ be a bounded sequence (for instance, $\left\|b_{n}\right\| \leq C$ for every $\left.n \in \mathbb{N}\right)$ and let $\left(e_{n}\right) \subset C\left(K_{1}\right)$ be a sequence such that $\left\|\left(e_{n}\right)_{n}\right\|_{1}^{\omega} \leq 1$. Then, the sequence $\left(e_{n} \otimes b_{n}\right) \subset C\left(K_{1}\right) \hat{\otimes}_{\pi} C\left(K_{2}\right)$ verifies that

$$
\left\|\left(e_{n} \otimes b_{n}\right)_{n}\right\|_{2}^{w} \leq \sqrt{2} C \text {. }
$$

We state now our first result, which gives sufficient conditions for the projective tensor product to have a complemented copy of $\ell_{2}$.

Theorem 2.2. Let $E, F$ be Banach spaces such that $E$ contains $c_{0}$ and $F$ contains a $C(K)$ space $G$ containing $\ell_{1}$. Then $E \hat{\otimes}_{\pi} F$ contains a complemented copy of $\ell_{2}$.

Proof. The proof is a generalization of [9, Theorem 1]. Let $\left(x_{n}\right)_{n} \subset E$ be sequence equivalent to the canonical basis of $c_{0}$, and let $\left(x_{n}^{*}\right)_{n} \subset E^{*}$ be a sequence biorthogonal to $\left(x_{n}\right)_{n}$. Let also $q: G \longrightarrow \ell_{2}$ be a surjective operator. By Grothendieck's theorem $q$ is 2-summing, hence, according to [13, Theorem 4.15], $q$ can be extended to a quotient $\tilde{q}: F \longrightarrow \ell_{2}$. Let $\left(b_{n}\right)_{n} \subset G \subset F$ be a bounded sequence such that $\tilde{q}\left(b_{n}\right)=q\left(b_{n}\right)=e_{n}$, where $\left(e_{n}\right)$ is the canonical basis in $\ell_{2}$. According to Lemma 2.1 the sequence $\left(x_{n} \otimes b_{n}\right)_{n}$ is 2 -weak summing when considered in $c_{0} \hat{\otimes}_{\pi} G$. Therefore, since the natural operator $c_{0} \hat{\otimes}_{\pi} G \longrightarrow E \hat{\otimes}_{\pi} F$ is continuous, $\left(x_{n} \otimes b_{n}\right)$ is also 2weak summing when considered in $E \hat{\otimes}_{\pi} F$. Therefore (see for example [13, Proposition 2.2]) we can define an operator

$$
\theta: \ell_{2} \longrightarrow E \hat{\otimes}_{\pi} F
$$

by

$$
\theta\left(e_{n}\right)=x_{n} \otimes b_{n} .
$$

Defined this way $\theta$ is bounded by $K_{G}$ times a bound for the sequence $\left(b_{n}\right)_{n}$, where $K_{G}$ is Grothendieck's constant.

We also define an operator

$$
\varphi: E \hat{\otimes}_{\pi} F \longrightarrow \ell_{2}
$$

by

$$
\varphi(a \otimes b)=\left(x_{n}^{*}(a) q(b)_{n}\right)_{n} .
$$

It is easy to see that $\varphi$ is well defined, continuous, and that $\varphi \circ \theta$ is the identity map on $\ell_{2}$, which finishes the proof.

REMARK 2.3. Let us recall that, given $\lambda>1$, a Banach space $X$ is said to be an $\mathcal{L}_{\infty, \lambda}$ space if every finite dimensional subspace $Y \subset X$ is contained in a finite dimensional space $Z \subset X$ for which there exists an isomorphism $v: Z \longrightarrow \ell_{\infty}^{\operatorname{dim} Z}$ such that $\|v\|\left\|v^{-1}\right\|<\lambda$. We say that $X$ is an $\mathcal{L}_{\infty}$ space if it is an $\mathcal{L}_{\infty, \lambda}^{\infty}$ space for some $\lambda>1 . L_{\infty}(\mu)$ and $C(K)$ spaces are the basic examples of $\mathcal{L}_{\infty}$-spaces. Clearly, our previous Theorem remains true if $G$ is any $\mathcal{L}_{\infty}$ space. 
It has been recently shown in [3] that the projective tensor product of two $\mathrm{JB}^{*}$-triples fails the DPP if and only if at least one of them contains $\ell_{1}$. As an application of our previous result we prove that in that case, actually there is a complemented copy of $\ell_{2}$ in the projective tensor product. We recall first some known results.

Let $x$ be an element in a complex JB*-triple $E$, and denote by $E(x)$ the $\mathrm{JB}^{*}$-subtriple of $E$ generated by $x$. It is known that there exists a locally compact subset $S_{x}$ of $(0,+\infty)$ such that $S_{x} \cup\{0\}$ is compact and $E(x)$ is $\mathrm{JB}^{*}$-triple isomorphic to the $\mathrm{C}^{*}$-algebra $C_{0}\left(S_{x}\right)$, via a triple isomorphism $\Psi$, which satisfies $\Psi(x)(t)=t\left(t \in S_{x}\right)$ (cf. [21, 4.8], [22, 1.15] and [16]). The subset $S_{x}$ is called the triple spectrum of $x$.

It is well known that for every infinite dimensional $\mathrm{C}^{*}$-álgebra $A$ there exists an infinite sequence $\left(x_{n}\right)$ in $A^{+}$satisfying $x_{n} x_{m}=x_{m} x_{n}=0$ and $\left\|x_{n}\right\|=1$, for all $n \neq m$ (compare [20, 4.6.13]). Since the subtriple generated by a single element $x$ in a complex JB*-triple $E$ is isomorphic as $\mathrm{JB}^{*}$-triple (and hence isometric as Banach space) to a $\mathrm{C}^{*}$-algebra, we can conclude, from [11, Theorem 6] and [6, Proposition 4.5], that for every non-reflexive complex $\mathrm{JB}^{*}$-triple, $E$, there exists an infinite sequence $\left(x_{n}\right)$ in $E$ satisfying $L\left(x_{n}, x_{m}\right)=L\left(x_{m}, x_{n}\right)=0$ and $\left\|x_{n}\right\|=1$, for all $n \neq m$. It is well known that $\left(x_{n}\right)$ is equivalent to the basis of $c_{0}$ (compare, for example, $[19, \S 4]$ ). Therefore, we have:

Corollary 2.4. Let $E, F$ be $J B^{*}$-triples such that $E$ is not reflexive and $F$ contains $\ell_{1}$. Then $E \hat{\otimes}_{\pi} F$ contains a complemented copy of $\ell_{2}$.

The corresponding result in the setting of $\mathrm{C}^{*}$-algebras follows straightforwardly from the above Corollary.

Corollary 2.5. Let $A, B$ be two infinite dimensional $C^{*}$-algebras such that $B$ contains $\ell_{1}$. Then $A \hat{\otimes}_{\pi} B$ contains a complemented copy of $\ell_{2}$.

There is also a local version of Theorem 2.2.

Theorem 2.6. Let $E, F$ be Banach spaces each one of them containing an isomorphic copy of $\mathcal{L}_{\infty}$ spaces $G, H$. Then $E \hat{\otimes}_{\pi} F$ contains uniformly complemented copies of the $\ell_{2}^{n}$ 's.

Proof. Let us fix $n \in \mathbb{N}$. There exists an $n$-dimensional space $X \subset G$ such that $d\left(X ; \ell_{\infty}^{n}\right) \leq C$, where $C$ is a constant independent of $n$. In $X$ we consider the canonical basis $\left(x_{m}^{n}\right)_{m=1}^{n}$ and we consider a sequence $\left(x_{m}^{n *}\right)_{m=1}^{n} \subset$ $E^{*}$ biorthogonal to it.

We fix an $\epsilon>0$ and Dvoretzky's Theorem, assures the existence of a number $N \in \mathbb{N}$ such that $\ell_{2}^{n}$ is $(1+\epsilon)$-contained in $\ell_{1}^{N}$. Therefore, there exists a quotient $q_{n}: \ell_{\infty}^{N} \longrightarrow \ell_{2}^{n}$ and a constant $K$ such that, for every $1 \leq n \leq m$ there exists $b_{n}^{m} \in \ell_{\infty}^{N}$ with $\left\|b_{n}^{m}\right\| \leq K$ and $q_{n}\left(b_{n}^{m}\right)=e_{m}$, where $K$ is a constant independent of $n$ and $1 \leq n \leq m$. We consider a space 
$Y \subset H$ verifying $d\left(Y, \ell_{\infty}^{N}\right) \leq C$ where is again independent of $N$. We consider the quotient $\hat{q_{n}}: Y \longrightarrow \ell_{2}^{n}$ induced by $q_{n}$. The 2 -summing norm of $\hat{q_{n}}$ is bounded by $\left\|q_{n}\right\| K_{G} C(1+\epsilon)$ (independently of $n$ ) and hence applying again $\left[13\right.$, Theorem 4.15] $\hat{q_{n}}$ can be extended to a quotient $\tilde{q_{n}}: F \longrightarrow \ell_{2}^{n}$ with no greater norm. Now the proof proceeds as in Theorem 2.2.

Corollary 2.7. Let $E, F$ be non reflexive $J B^{*}$-triples. Then $E \hat{\otimes}_{\pi} F$ contains uniformly complemented copies of the $\ell_{2}^{n}$ 's. In particular, the result remains true whenever $E$ and $F$ are infinite dimensional $C^{*}$-algebras.

We will substantially improve this last corollary in our next result.

In [3, Remark 2.7], the authors prove the existence of JB*-triples $E, F$ such that $E \hat{\otimes}_{\pi} F$ has DPP, $\left(E \hat{\otimes}_{\pi} F\right)^{*}$ is a Schur space and $\left(E \hat{\otimes}_{\pi} F\right)^{* *}$ does not have DPP.

Until quite recently, the (essentially) unique example of a space $X$ with the DPP, such that $X^{*}$ does not have DPP was Stegall's example $c_{0}\left(\ell_{2}^{n}\right)$; $\left(c_{0}\left(\ell_{2}^{n}\right)\right)^{*}=\ell_{1}\left(\ell_{2}^{n}\right)$ is a Schur space, but $\left(c_{0}\left(\ell_{2}^{n}\right)\right)^{* *}=\ell_{\infty}\left(\ell_{2}^{n}\right)$ contains a complemented copy of $\ell_{2}$, and therefore does not have DPP [25].

Recently it was known that the same situation happens with $c_{0} \hat{\otimes}_{\pi} c_{0}$ : that is, $\left(c_{0} \hat{\otimes}_{\pi} c_{0}\right)^{*}$ is a Schur space but $\left(c_{0} \hat{\otimes}_{\pi} c_{0}\right)^{* *}$ does not have DPP $([17,8])$. Very recently, it was proved in [9] that $c_{0} \hat{\otimes}_{\pi} c_{0}$ contains a complemented copy of $c_{0}\left(\ell_{2}^{n}\right)$.

We prove next that the above mentioned projective tensor product of $\mathrm{JB}^{*}$-triples appearing in [3] contains a complemented copy of $c_{0} \hat{\otimes}_{\pi} c_{0}$, and hence it also contains a complemented copy of $c_{0}\left(\ell_{2}^{n}\right)$. Therefore, Stegall's example remains essentially unique.

Other interesting examples of JB*-triples are constituted by the so-called Cartan factors, $C_{\alpha}(\alpha=1, \ldots, 6)$, defined as follows: let $H$ and $K$ be complex Hilbert spaces and let $j: H \rightarrow H$ be a conjugation (conjugate linear isometry of period-2) on $H, C_{1}=L(H, K), C_{2}=\left\{x \in L(H): j x^{*} j=\right.$ $-x\}, C_{3}=\left\{x \in L(H): j x^{*} j=x\right\}, C_{4}$ is a complex spin factor (that is, a renormed Hilbert space, see for example [3, Page 9]), $C_{5}=M_{1,2}(\mathbb{O})$, and $C_{6}=H_{3}(\mathbb{O})$, the hermitian $3 \times 3$ matrices with entries in the eightdimensional Cayley division algebra $\mathbb{O}$. The elementary JB*-triples, $K_{\alpha}$ $(\alpha=1, \ldots, 6)$, introduced in [5, page 330], can be described in the following way: $K_{1}=K(H, K), K_{\alpha}=C_{\alpha} \cap K(H)$, for $\alpha=2,3$, and $K_{\alpha}=C_{\alpha}$, for $\alpha=4,5,6$.

The following result, which is needed to prove Corollary 2.9, is probably interesting by itself.

Theorem 2.8. Let $E$ be a not reflexive $J B^{*}$-triple non containing $\ell_{1}$. Then $E$ contains a complemented copy of $c_{0}$.

Proof. Let $A$ denote the weak*-closed triple ideal generated by all minimal tripotents of $E^{* *}$, and let $K_{0}(E)=E \cap A$. It is known that $K_{0}(E)$ is an 
inner ideal of $E$ (compare [6, Corollary 3.5]). It is also known that $K_{0}(E)$ is a $c_{0}$-sum of a family, $\left\{K_{i}\right\}_{i \in \Lambda}$, of elementary $\mathrm{JB}^{*}$-triples [6, Lemma 3.3].

It is known that a $\mathrm{JB}^{*}$-triple satisfies the RNP if and only if it is reflexive (compare [11, Theorem 6]). Now it follows from [5, Theorem 3.4] and [6, Proposition 4.5] that the triple spectrum of every element $x \in E$ is countable and there exists at least one $x$ in $E$ whose triple spectrum, $S_{x}$, must be infinite. Arguing as in the proof of [5, Theorem 3.4, (iv) $\Rightarrow(v)]$ (see also the proof of [5, Proof of Lemma 3.2], we deduce that, for each $n \in \mathbb{N}, E$ contains a set $\left\{e_{1}, \ldots, e_{n}\right\}$ of pairwise orthogonal minimal tripotents of $E^{* *}$. In particular, we have $K_{0}(E) \neq\{0\}$. ¿From [2, Lemma 3.7 and Theorem $3.8]$ we deduce that there exists a sequence $\left(e_{n}\right) \subset E$ of pairwise orthogonal tripotents of $E^{* *}$.

Therefore, there exists an inner ideal $I$ of $E$ containing a sequence $\left(e_{n}\right)$ of pairwise orthogonal tripotents of $E^{* *}$. The series $\sum_{n} e_{n}$ is weak*-convergent to a tripotent $u \in I^{* *}$ (compare [19]). Since for each $x \in I$, the series $\sum_{n \in \mathbb{N}} P_{2}\left(e_{n}\right)(x)$ converges in the weak*-topology of $I^{* *}$ to $P_{2}(u)(x)$ we deduce that the mapping $P: I \rightarrow \operatorname{Lin}\left\{e_{n}\right\}=c_{0}, P(x):=\left(P_{2}\left(e_{n}\right)(x)\right)_{n \in \mathbb{N}}$ is a bounded linear projection of $I$ onto $c_{0}$. Since $I$ is an inner ideal of $E$, then for each $x \in E$ and $n \in \mathbb{N}$, we have $P_{2}\left(e_{n}\right)(x)=\left\{e_{n},\left\{e_{n}, x, e_{n}\right\}, e_{n}\right\} \in I$ and hence $\left(P_{2}\left(e_{n}\right)(x)\right)_{n}=\left(P_{2}\left(e_{n}\right) P_{2}\left(e_{n}\right)(x)\right)_{n} \in c_{0}$. Therefore, $P: E \rightarrow c_{0}$ is a well-defined contractive projection of $E$ onto $c_{0}$.

The case $E=\ell_{\infty}$ tells us that we can not just remove the condition that $E$ does not contain $\ell_{1}$ in the above theorem.

The following announced corollary follows now immediately.

Corollary 2.9. Let $E$ and $F$ be two $J B^{*}$-triples non containing $\ell_{1}$ and satisfying the DPP. Then $E \hat{\otimes}_{\pi} F$ contains a complemented subspace isomorphic to $c_{0} \hat{\otimes}_{\pi} c_{0}$. As a consequence, $E \hat{\otimes}_{\pi} F$ contains a complemented subspace isomorphic to $c_{0}\left(\ell_{2}^{n}\right)$.

Proof. We just need to observe that reflexive Banach spaces never have DPP. The last statement follows now from [9], where it is proved that $c_{0} \hat{\otimes}_{\pi} c_{0}$ contains a complemented copy of $c_{0}\left(\ell_{2}^{n}\right)$.

\section{The alternative Dunford-Pettis Property on tensor PRODUCTS}

In this section we find sufficient conditions to assure that the projective tensor product of two Banach spaces does not have DP1. These conditions will completely determine those $\mathrm{C}^{*}$-algebras whose projective tensor product satisfies DP1. They also solve the same problem for many, but not all, cases of $\mathrm{JB}^{*}$-triples.

We develop first some necessary tools. 
Let $X_{1}, X_{2}, Y_{1}, Y_{2}$ be real Banach spaces. Every bounded linear operator $T$ from $X_{1} \oplus^{\ell_{\infty}} X_{2}$ to $Y_{1} \oplus^{\ell_{1}} Y_{2}$, can be written as a matrix

$$
T=\left(\begin{array}{ll}
T_{1} & T_{2} \\
T_{3} & T_{4}
\end{array}\right)
$$

where $T_{1}: X_{1} \rightarrow Y_{1}, T_{2}: X_{2} \rightarrow Y_{1}, T_{3}: X_{1} \rightarrow Y_{2}$, and $T_{4}: X_{2} \rightarrow Y_{2}$ are bounded linear operators.

Lemma 3.1. Let $X_{1}, X_{2}, Y_{1}, Y_{2}$ be Banach spaces. The law

$$
T=\left(\begin{array}{cc}
T_{1} & T_{2} \\
T_{3} & T_{4}
\end{array}\right) \mapsto P(T)=\left(\begin{array}{cc}
T_{1} & 0 \\
0 & T_{4}
\end{array}\right),
$$

defines a contractive linear projection on $L\left(X_{1} \oplus^{\ell_{\infty}} X_{2}, Y_{1}^{*} \oplus^{\ell_{1}} Y_{2}^{*}\right)$. Moreover, $\|P(T)\|=\left\|T_{1}\right\|+\left\|T_{4}\right\|$.

Proof. Suppose first that $X_{1}, X_{2}, Y_{1}$ and $Y_{2}$ are real Banach spaces. Let $T \in L\left(X_{1} \oplus^{\ell_{\infty}} X_{2}, Y_{1}^{*} \oplus^{\ell_{1}} Y_{2}^{*}\right)$.

$$
\begin{gathered}
\quad\|T\|=\sup _{\left\|x_{i}\right\| \leq 1}\left\|T\left(x_{1}, x_{2}\right)\right\|=\sup _{x_{i} \in B_{X_{i}} \varphi_{i} \in B_{Y_{i}^{*}}}\left|\left(\varphi_{1}+\varphi_{2}\right) T\left(x_{1}+x_{2}\right)\right| \\
=\sup _{x_{i} \in B_{X_{i}} \varphi_{i} \in B_{Y_{i}^{*}}} \sup _{1}\left|\varphi_{1} T_{1}\left(x_{1}\right)+\varphi_{1} T_{2}\left(x_{2}\right)+\varphi_{2} T_{3}\left(x_{1}\right)+\varphi_{2} T_{4}\left(x_{2}\right)\right| \\
=\sup _{x_{i} \in B_{X_{i}}} \sup _{\varphi_{i} \in B_{Y_{i}^{*}}}\left|\varphi_{1} T_{1}\left(x_{1}\right)+\varphi_{1} T_{2}\left(x_{2}\right)\right|+\left|\varphi_{2} T_{3}\left(x_{1}\right)+\varphi_{2} T_{4}\left(x_{2}\right)\right| \\
=\sup _{x_{i} \in B_{X_{i}}, \varphi_{i} \in B_{Y_{i}^{*}}} \max \left\{\left|\varphi_{1} T_{1}\left(x_{1}\right)+\varphi_{1} T_{2}\left(x_{2}\right)\right|+\left|\varphi_{2} T_{3}\left(x_{1}\right)+\varphi_{2} T_{4}\left(x_{2}\right)\right|,\right. \\
=\sup _{x_{i} \in B_{X_{i}}, \varphi_{i} \in B_{Y_{i}^{*}}} \max \left\{\left|\varphi_{1} T_{1}\left(x_{1}\right)+\varphi_{1} T_{2}\left(x_{2}\right)+\varphi_{2} T_{3}\left(x_{1}\right)+\varphi_{2} T_{4}\left(x_{2}\right)\right|,\right. \\
\left|\varphi_{1} T_{1}\left(x_{1}\right)+\varphi_{1} T_{2}\left(x_{2}\right)-\varphi_{2} T_{3}\left(x_{1}\right)-\varphi_{2} T_{4}\left(x_{2}\right)\right|, \mid \varphi_{1} T_{1}\left(x_{1}\right)-\varphi_{1} T_{2}\left(x_{2}\right) \\
\left.+\varphi_{2} T_{3}\left(x_{1}\right)-\varphi_{2} T_{4}\left(x_{2}\right)|,| \varphi_{1} T_{1}\left(x_{1}\right)-\varphi_{1} T_{2}\left(x_{2}\right)-\varphi_{2} T_{3}\left(x_{1}\right)+\varphi_{2} T_{4}\left(x_{2}\right) \mid\right\} \\
=\sup _{x_{i} \in B_{X_{i}}, \varphi_{i} \in B_{Y_{i}^{*}}} \max \left\{\left|\varphi_{1} T_{1}\left(x_{1}\right)+\varphi_{2} T_{4}\left(x_{2}\right)\right|+\left|\varphi_{1} T_{2}\left(x_{2}\right)+\varphi_{2} T_{3}\left(x_{1}\right)\right|\right. \\
\geq \\
\sup _{x_{i} \in B_{X_{i}}, \varphi_{i} \in B_{Y_{i}^{*}}} \max \left\{\left|\varphi_{1} T_{1}\left(x_{1}\right)-\varphi_{2} T_{4}\left(x_{2}\right)\right|+\left|\varphi_{1} T_{2}\left(x_{2}\right)-\varphi_{2} T_{3}\left(x_{1}\right)\right|\right\} \\
=\sup _{\left.x_{i} \in B_{X_{i}}, \varphi_{i} \in B_{Y_{i}^{*}} T_{4}\left(x_{2}\right)|,| \varphi_{1} T_{1}\left(x_{1}\right)-\varphi_{2} T_{4}\left(x_{2}\right) \mid\right\}}\left|\varphi_{1} T_{1}\left(x_{1}\right)\right|+\left|\varphi_{2} T_{4}\left(x_{2}\right)\right|=\left\|T_{1}\right\|+\left\|T_{4}\right\|=\mid T_{1}+T_{4} \| .
\end{gathered}
$$

When $X_{1}, X_{2}, Y_{1}$ and $Y_{2}$ are complex Banach spaces we can apply the formula

$$
\max _{\theta \in[0,2 \pi]}\left\{\left|e^{i \theta} a+b\right|,\left|e^{i \theta} a-b\right|\right\}=|a|+|b|,
$$

$(a, b \in \mathbb{C}$,$) instead of |a|+|b|=\max \{|a+b|,|a-b|\},(a, b \in \mathbb{R}$,$) to get the$ statement in the complex case. 
We need the following proposition, which might be useful also in different settings.

Proposition 3.2. Let $X_{1}, X_{2}, Y_{1}$ and $Y_{2}$ be Banach spaces. Then $\left(X_{1} \hat{\otimes}_{\pi} Y_{1}\right) \oplus^{\ell_{\infty}}\left(X_{2} \hat{\otimes}_{\pi} Y_{2}\right)$ is complemented in $\left(X_{1} \oplus^{\ell_{\infty}} X_{2}\right) \hat{\otimes}_{\pi}\left(Y_{1} \oplus^{\ell_{\infty}} Y_{2}\right)$.

Proof. Let $p_{1}$ and $p_{2}$ denote the contractive projections of $X_{1} \oplus^{\ell_{\infty}} X_{2}$ and $Y_{1} \oplus^{\ell \infty} Y_{2}$ onto $X_{1}$ and $Y_{1}$, respectively, and let $q_{1}=1-p_{1}$ and $q_{2}=1-p_{2}$. By [12, Proposition 3.9] we conclude that the mapping $\Pi=p_{1} \otimes p_{2}+q_{1} \otimes q_{2}$ is a bounded linear projection on $\left(X_{1} \oplus^{\ell_{\infty}} X_{2}\right) \hat{\otimes}_{\pi}\left(Y_{1} \oplus^{\ell_{\infty}} Y_{2}\right),\|\Pi\| \leq 2$ and

$$
\|\Pi(\alpha)\|=\left\|p_{1} \otimes p_{2}(\alpha)+q_{1} \otimes q_{2}(\alpha)\right\| \geq \max \left\{\left\|p_{1} \otimes p_{2}(\alpha)\right\|,\left\|q_{1} \otimes q_{2}(\alpha)\right\|\right\} .
$$

We claim that, in fact

$$
\|\Pi(\alpha)\|=\left\|p_{1} \otimes p_{2}(\alpha)+q_{1} \otimes q_{2}(\alpha)\right\|=\max \left\{\left\|p_{1} \otimes p_{2}(\alpha)\right\|,\left\|q_{1} \otimes q_{2}(\alpha)\right\|\right\} .
$$

Indeed, let us denote $\alpha_{1}=p_{1} \otimes p_{2}(\alpha)$ and $\alpha_{2}=q_{1} \otimes q_{2}(\alpha)$.

$$
\begin{gathered}
\left\|\alpha_{1}+\alpha_{2}\right\|=\sup _{T \in B_{L\left(X_{1} \oplus^{\infty} X_{2}, Y_{1}^{*} \oplus^{1} Y_{2}^{*}\right)}}\left|T\left(\alpha_{1}\right)+T\left(\alpha_{2}\right)\right| \\
=\sup _{1 \geq\|T\| \geq\left\|T_{1}\right\|+\left\|T_{4}\right\|}\left|T_{1}\left(\alpha_{1}\right)+T_{4}\left(\alpha_{2}\right)\right| \leq \sup _{1 \geq\left\|T_{1}\right\|+\left\|T_{4}\right\|}\left|T_{1}\left(\alpha_{1}\right)\right|+\left|T_{4}\left(\alpha_{2}\right)\right| \\
=\max \left\{\left\|\alpha_{1}\right\|,\left\|\alpha_{2}\right\|\right\} .
\end{gathered}
$$

Let $X$ be a Banach space. We say that two elements $x, y \in X$ are Morthogonal if and only if $\|\lambda x+\mu y\|=\max \{\|\lambda x\|,\|\mu y\|\}$, for all $\lambda, \mu \in \mathbb{K}$.

Corollary 3.3. Let $X$ and $Y$ be Banach spaces. Let $x_{1}, x_{2} \in X$ and $y_{1}, y_{2} \in$ $Y$ be such that $x_{1}$ is $M$-orthogonal to $x_{2}$ and $y_{1}$ is $M$-orthogonal to $y_{2}$. Then, if $\|\cdot\|_{\pi}$ denotes the projective tensor norm in $X \hat{\otimes}_{\pi} Y$, we have $\| x_{1} \otimes y_{1}+x_{2} \otimes$ $y_{2} \|_{\pi}=\max \left\{\left\|x_{1} \otimes y_{1}\right\|_{\pi},\left\|x_{2} \otimes y_{2}\right\|_{\pi}\right\}$.

Proof. Let $A, B$ be Banach spaces. Following the notation in [12], in this proof the projective tensor norm of an element $\alpha \in A \hat{\otimes}_{\pi} B$ will also be denoted by $\pi(\alpha ; A, B)$. From [12, 3.2.(5)], we conclude that

$$
\pi(\alpha ; X, Y)=\inf \left\{\pi(\alpha ; M, N): \begin{array}{l}
\alpha \in M \otimes N ; M \subset X \text { and } \\
N \subset Y \text { finite dimensional }
\end{array}\right\} .
$$

Let $M$ (respectively, $N$ ) denote the linear span of $x_{1}$ and $x_{2}$ (respectively, $y_{1}$ and $\left.y_{2}\right)$. Clearly, $M=\mathbb{K} x_{1} \oplus^{\infty} \mathbb{K} x_{2}$ and $N=\mathbb{K} y_{1} \oplus^{\infty} \mathbb{K} y_{2}$. ¿From (2) we have

$\left\|x_{1} \otimes y_{1}+x_{2} \otimes y_{2}\right\|_{\pi}=\pi\left(x_{1} \otimes y_{1}+x_{2} \otimes y_{2} ; X, Y\right) \leq \pi\left(x_{1} \otimes y_{1}+x_{2} \otimes y_{2} ; M, N\right)$.

Now Proposition 3.2 gives that

$$
\begin{gathered}
\pi\left(x_{1} \otimes y_{1}+x_{2} \otimes y_{2} ; M, N\right)=\max \left\{\pi\left(x_{1} \otimes y_{1} ; M, N\right), \pi\left(x_{2} \otimes y_{2} ; M, N\right)\right\} \\
=\max \left\{\left\|x_{1} \otimes y_{1}\right\|_{\pi},\left\|x_{2} \otimes y_{2}\right\|_{\pi}\right\},
\end{gathered}
$$


showing that

$$
\left\|x_{1} \otimes y_{1}+x_{2} \otimes y_{2}\right\|_{\pi} \leq \max \left\{\left\|x_{1} \otimes y_{1}\right\|_{\pi},\left\|x_{2} \otimes y_{2}\right\|_{\pi}\right\} .
$$

To see the reverse inequality, suppose that $\max \left\{\left\|x_{1} \otimes y_{1}\right\|_{\pi},\left\|x_{2} \otimes y_{2}\right\|_{\pi}\right\}=$ $\left\|x_{1} \otimes y_{1}\right\|_{\pi}$. Let $\varphi$ be the norm-one functional in $M^{*}$ given by $\varphi\left(\lambda x_{1}+\mu x_{2}\right)=$ $\lambda\left\|x_{1}\right\|$, and let $\phi \in X^{*}$ a Hahn-Banach extension of $\varphi$. For every $\varepsilon>0$ we can take a norm-one functional $\psi \in Y^{*}$ such that $\psi\left(y_{1}\right)>\left\|y_{1}\right\|-\varepsilon$. Let $T \in L\left(X, Y^{*}\right)$ be the norm-one linear operator defined by $T(x)(y):=$ $\phi \otimes \psi(x \otimes y)=\phi(x) \psi(y)$. The it follows that

$$
\left\|x_{1} \otimes y_{1}+x_{2} \otimes y_{2}\right\|_{\pi} \geq\left|T\left(x_{1} \otimes y_{1}+x_{2} \otimes y_{2}\right)\right|=\left\|x_{1}\right\|\left(\left\|y_{1}\right\|-\varepsilon\right),
$$

which gives the desired inequality.

REMARK 3.4. In $C[0,1]$ we can always find a norm one operator $q: C[0,1] \longrightarrow \ell_{2}$ and a sequence, $\left(y_{n}\right)$, in the unit sphere of $C[0,1]$ such that $q\left(y_{n}\right)=\frac{1}{\sqrt{2}} e_{n}$, where $\left(e_{n}\right)_{n} \subset \ell_{2}$ is the canonical basis. One way to do this is the following: we consider $\lambda$ to be the Lebesgue measure on $[0,1]$. The law

$$
\langle f, g\rangle=\int_{0}^{1} f \bar{g} d \lambda, \quad f, g \in C[0,1],
$$

defines a positive sesquilinear form on $C[0,1]$ with prehilbertian norm denoted by $\|\cdot\|_{\lambda}$. Then the completion $H_{\lambda}$ of $\left(C[0,1],\|\cdot\|_{\lambda}\right)$ is a Hilbert space with respect to the norm $\|\cdot\|_{\lambda}$. The natural inclusion $q:\left(C[0,1],\|\cdot\|_{\infty}\right) \longrightarrow H_{\lambda}$ is clearly a norm one linear operator and defining $y_{n}(t):=\sin (2 \pi n t)(t \in[0,1], n \in \mathbb{N})$ we get the desired statement.

We prove now the main result of the paper.

Theorem 3.5. Let $E, F$ be two Banach spaces such that $E$ contains an isometric copy of $c_{0}$ and $F$ contains and isometric copy of $C[0,1]$. Then $E \hat{\otimes}_{\pi} F$ does not have DP1.

Proof. We first observe that $C[0,1]$ contains $C\left(\left[0, \frac{1}{4}\right] \cup\left[\frac{3}{4}, 1\right]\right)=C\left[0, \frac{1}{4}\right] \oplus_{\infty}$ $C\left[\frac{3}{4}, 1\right]$ isometrically and 1-complemented, with the inclusion

$$
i: C\left(\left[0, \frac{1}{4}\right] \cup\left[\frac{3}{4}, 1\right]\right) \longrightarrow C[0,1]
$$

given by

$$
i(f, g)(t)=\left\{\begin{array}{cc}
f(t) & \text { if } t \in\left[0, \frac{1}{4}\right] \\
0 & \text { if } t=\frac{1}{2} \\
g(t) & \text { if } t \in\left[\frac{3}{4}, 1\right] \\
\text { linear in }\left(\frac{1}{4}, \frac{1}{2}\right) \text { and }\left(\frac{1}{2}, \frac{3}{4}\right) &
\end{array}\right.
$$


and the natural proyection

$$
\pi: C[0,1] \longrightarrow C\left(\left[0, \frac{1}{4}\right] \cup\left[\frac{3}{4}, 1\right]\right)
$$

given by the restriction, that is,

$$
\pi(f)(t)=f(t) .
$$

We consider the function $\mathbb{1}:\left[0, \frac{1}{4}\right] \longrightarrow \mathbb{K}$ of constant value 1 , and we define $y_{0}=i(\mathbb{1}) \in C[0,1]$. Reasoning as in Remark 3.4 we can also consider a sequence $\left(z_{n}\right)_{n} \subset C\left[\frac{3}{4}, 1\right]$ of norm one functions and a mapping

$$
p: C\left[\frac{3}{4}, 1\right] \longrightarrow \ell_{2}
$$

such that $p\left(z_{n}\right)=\frac{1}{\sqrt{2}} e_{n}$. For every $n \in \mathbb{N}$, we define $y_{n}=i\left(z_{n}\right)$. Then $\left\|y_{n}\right\|=$ 1 for every $n \in \mathbb{N}$. It follows from the definitions that, for every $n \in \mathbb{N}, y_{0}$ is M-orthogonal to $y_{n}$. We can also consider the operator $q: C[0,1] \longrightarrow \ell_{2}$ which naturally extends $p$, that is, $q=p \circ \pi_{2}$, where $\pi_{2}: C[0,1] \longrightarrow C\left[\frac{3}{4}, 1\right]$ is again the projection induced by the restriction.

We consider now the sequence $\left(e_{n} \otimes y_{n}\right)_{n \geq 1} \subset E \hat{\otimes}_{\pi} F$. Clearly

$$
\left\|e_{n} \otimes y_{n}\right\|_{E \hat{\otimes}_{\pi} F}=\left\|e_{n}\right\|\left\|y_{n}\right\|=1 .
$$

Considering $c_{0}$ as $c_{0}(\mathbb{N} \cup\{0\})$, we consider the vector $e_{0} \otimes y_{0} \in E \hat{\otimes}_{\pi} F$, which also has norm 1.

An application of Corollary 3.3 shows that

$$
\left\|e_{n} \otimes y_{n}+e_{0} \otimes y_{0}\right\|_{E \hat{\otimes}_{\pi} F}=1 \text {. }
$$

It follows from Grothendieck's theorem that $q: C[0,1] \longrightarrow \ell_{2}$ is 2summing. Therefore, [13, Theorem 4.15] allows us to extend $q$ to an operator $\tilde{q}: F \longrightarrow \ell_{2}$. We consider now a sequence $\left(x_{n}^{*}\right) \in E^{*}$ biorthogonal to the $c_{0}$-basis $\left(e_{n}\right) \subset E$ and we define the operator

$$
\theta: E \hat{\otimes}_{\pi} F \longrightarrow \ell_{2}
$$

by

$$
\theta(a \otimes b)=\left(x_{n}^{*}(a) \tilde{q}(b)_{n}\right)_{n \geq 1}
$$

It is not difficult to see that $\theta$ is well defined and continuous (the easiest way is to consider $\theta$ as a bilinear operator on $E \times F)$.

We consider now the sequence $\left(\theta^{*}\left(e_{n}\right)\right)_{n} \subset\left(E \hat{\otimes}_{\pi} F\right)^{*}$, where $\left(e_{n}\right)_{n} \subset \ell_{2}$ denotes now the canonical basis of $\ell_{2}$. Clearly $\left(\theta^{*}\left(e_{n}\right)\right)_{n}$ weakly converges to 0 .

Finally, noticing that

$$
\theta\left(e_{0} \otimes y_{0}\right)=0
$$

(both because $x_{n}^{*}\left(e_{0}\right)=0$ for every $n \geq 1$ and because $\tilde{q}\left(y_{0}\right)=q\left(y_{0}\right)=$ $\left.p\left(\pi_{2}\right)\left(y_{0}\right)=p(0)=0\right)$ we have

$$
\theta^{*}\left(e_{n}\right)\left(e_{n} \otimes y_{n}+e_{0} \otimes y_{0}\right)=\theta^{*}\left(e_{n}\right)\left(e_{n} \otimes y_{n}\right)+\theta^{*}\left(e_{n}\right)\left(e_{0} \otimes y_{0}\right)=
$$




$$
=\theta\left(e_{n} \otimes y_{n}\right)_{n}+\theta\left(e_{0} \otimes y_{0}\right)_{n}=\frac{1}{\sqrt{2}}+0 \nrightarrow 0 .
$$

Next we specialize the last result for the case of JB*-triples.

Corollary 3.6. Let $E, F$ be $J B^{*}$-triples such that $E$ is not reflexive and $F$ contains $\ell_{1}$. Then $E \hat{\otimes}_{\pi} F$ does not have DP1.

Proof. By the remarks preceding Corollary 2.4, we know that $E$ contains an isometric copy of $c_{0}$.

Moreover, it is known that a $\mathrm{JB}^{*}$-triple $F$ contains a subspace isomorphic to $\ell_{1}$ if and only there exists a norm-one element $x \in F$ such that the triple spectrum of $x$ is not countable [5, Theorem 3.4]. Therefore, we may assume that there exists a norm-one element $x \in F$ such that $S_{x}$ is not countable. We recall that $1 \in S_{x}$ is a locally compact subset of $(0,1], S_{x} \cup\{0\}$ is compact and the $\mathrm{JB}^{*}$-subtriple of $F$ generated by $x$ is triple isomorphic (and hence isometric) to $C_{0}\left(S_{x}\right)$ (the Banach space of all complex-valued continuous functions on $S_{x}$ vanishing at 0$)$.

It is clear that $C_{0}\left(S_{x}\right)$ is also a JB*-subtriple of $C\left(S_{x} \cup\{0\}\right)$. Since $S_{x}$ is not countable, it follows, again from [5, Theorem 3.4], that $C_{0}\left(S_{x}\right)$ and hence $C\left(S_{x} \cup\{0\}\right)$ contains a subspace isomorphic to $\ell_{1}$. From [23] we conclude that $S_{x} \cup\{0\}$ is non scattered and thus there exists a continuous surjection $\sigma: S_{x} \cup\{0\} \rightarrow[0,1]$ (compare [24, 8.5.4]).

The mapping $\Phi: C([0,1]) \rightarrow C\left(S_{x} \cup\{0\}\right)$ defined by $\Phi(f)(t):=f(\sigma(t))$ is an isometric linear JB*-triple embedding of $C([0,1])$ into $C\left(S_{x} \cup\{0\}\right)$. If we denote $\sigma(0)=a \in[0,1]$ and $C_{0}([0,1] \backslash\{a\})$ the Banach space of all complex-valued continuous functions on $[0,1] \backslash\{a\}$ vanishing at $a$, it follows that $\left.T\right|_{C_{0}([0,1] \backslash\{a\})}$ is an isometric linear triple embedding of $C_{0}([0,1] \backslash\{a\})$ into $C_{0}\left(S_{x} \cup\{0\}\right)$. Since $C_{0}([0, a))$ or $C_{0}((a, 1])$ is triple isomorphic (an hence isometric) to $C_{0}((0,1])$ we may always assume that there exists a isometric linear JB*-triple embedding of $C_{0}((0,1])$ into $C_{0}\left(S_{x} \cup\{0\}\right)$ and hence into $F$. Therefore, we may assume, without lost of generality, that $C_{0}((0,1])$ is a JB*-subtriple of $F$.

The result follows now from our previous theorem.

We have already commented that every infinite dimensional $\mathrm{C}^{*}$-algebra is not reflexive, in fact, both properties are equivalent. In [7] it is shown that the DPP and the DP1 are equivalent in every $\mathrm{C}^{*}$-algebra. Combining the above comments with Theorem 3.6 and [3, Corollary 2.5] we get the following.

Corollary 3.7. Let $A$ and $B$ be infinite dimensional $C^{*}$-álgebras. Then the following are equivalent:

(a) $A \hat{\otimes}_{\pi} B$ satisfies the DP1;

(b) $A$ and $B$ satisfy the DPP and do not contain $\ell_{1}$;

(c) $A \hat{\otimes}_{\pi} B$ satisfies the DPP; 
Corollary 3.8. Let $K_{1}$ and $K_{2}$ be infinite compact Hausdorff spaces. Then the following are equivalent:

(a) $C\left(K_{1}\right) \hat{\otimes}_{\pi} C\left(K_{2}\right)$ satisfies the DP1;

(b) $C\left(K_{1}\right)$ and $C\left(K_{2}\right)$ satisfy the DPP and do not contain $\ell_{1}$;

(c) $C\left(K_{1}\right) \hat{\otimes}_{\pi} C\left(K_{2}\right)$ satisfies the DPP;

Problem 3.9. In order to determine when the projective tensor product of two JB*-triples satisfies DP1, we need to know whether $E \hat{\otimes}_{\pi} F$ has DP1, when only one of them is reflexive. In particular, we do not know whether $c_{0} \hat{\otimes}_{\pi} \ell_{2}$ or $C[0,1] \hat{\otimes}_{\pi} \ell_{2}$ have DP1. In both cases we have DPP in one of the factors and the Kadec-Klee Property in the other one.

\section{REFERENCES}

[1] Acosta, M. D. and Peralta, A. M., An alternative Dunford-Pettis property for JB*triples, Quart. J. Math. Oxford Ser. 52, 391-401 (2001).

[2] Becerra Guerrero, J., López Pérez, G., Peralta, A. M. and Rodríguez Palacios, A., Relatively weakly open sets in closed balls of Banach spaces, and real JB*-triples of finite rank, Math. Ann. 330, 45-58 (2004).

[3] Becerra Guerrero, J. and Peralta, A. M., The Dunford-Pettis and the Kadec-Klee properties on tensor products of $\mathrm{JB}^{*}$-triples, to appear in Math. Z.

[4] Bombal, F. and Villanueva, I., On the Dunford-Pettis property of the tensor product of $C(K)$ spaces, Proc. Amer. Math. Soc. 129, no. 5, 1359-1363 (2001).

[5] Bunce, L. J. and Chu, Ch.-H., Dual spaces of JB*-triples and the Radon-Nikodým property, Math. Z. 208, no. 2, 327-334 (1991).

[6] Bunce, L. J. and Chu, Ch.-H., Compact operations, multipliers and Radon-Nikodým property in JB*-triples, Pacific J. Math. 153, no. 2, 249-265 (1992).

[7] Bunce, L. J. and Peralta, A. M., The alternative Dunford-Pettis property in C*algebras and von Neumann preduals, Proc. Amer. Math. Soc. 131, No. 4, 1251-1255 (2002).

[8] F. Cabello and R. García, The bidual of a tensor product of Banach spaces. BUSCARLO

[9] Cabello, F., Pérez-García, D. and Villanueva, I., Unexpected subspaces of tensor products, preprint 2004

[10] Chu, Ch.-H. and Iochum, B., On the Radon-Nikodým property in Jordan triples, Proc. Amer. Math. Soc. 99, no. 3, 462-464 (1987).

[11] Chu, Ch.-H. and Iochum, B., Complementation of Jordan triples in von Neumann algebras, Proc. Amer. Math. Soc. 108, no. 1, 19-24 (1990).

[12] Defant, A. and Floret, K., Tensor norms and operator ideals, North-Holland Mathematics Studies, 176. North-Holland Publishing Co., Amsterdam, 1993.

[13] J. Diestel, H. Jarchow and A. Tonge, Absolutely Summing Operators. Cambridge Univ. Press, 1995.

[14] Dineen, S., The second dual of a JB*-triple system, In: Complex analysis, functional analysis and approximation theory (ed. by J. Múgica), 67-69, (North-Holland Math. Stud. 125), North-Holland, Amsterdam-New York, 1986.

[15] Freedman, W., An alternative Dunford-Pettis property, Studia Math. 125, 143-159 (1997).

[16] Friedman, Y. and Russo, B., Structure of the predual of a JBW*-triple, J. Reine u. Angew. Math. 356, 67-89 (1985).

[17] González, M. and Gutiérrez, J., The Dunford-Pettis property on tensor products, Math. Proc. Camb. Phil. Soc., 131 (2001), 185-192. 
[18] Grothendieck, A., Sur les applications lineaires faiblement compactes d'espaces du type $C(K)$, Canad. J. Math. 5 129-173 (1953).

[19] Horn, G., Characterization of the predual and ideal structure of a JBW*-triple, Math. Scand. 61, no. 1, 117-133 (1987).

[20] Kadison, R. V. and Ringrose, J. R., Fundamentals of the theory of operator algebras. Vol. I. Elementary theory. Pure and Applied Mathematics, 100. Academic Press, Inc. [Harcourt Brace Jovanovich, Publishers], New York, 1983.

[21] Kaup, W., Algebraic Characterization of symmetric complex Banach manifolds, Math. Ann. 228, 39-64 (1977).

[22] Kaup, W., A Riemann Mapping Theorem for bounded symmentric domains in complex Banach spaces, Math. Z. 183, 503-529 (1983).

[23] Pełczyński, A. and Semadeni, Z., Spaces of continuous functions. III. Spaces $C(\Omega)$ for $\Omega$ without perfect subsets, Studia Math. 18, 211-222 (1959).

[24] Semadeni, Z., Banach spaces of continuous functions. Vol. I. Monografie Matematyczne, Tom 55. PWN-Polish Scientific Publishers, Warsaw, 1971.

[25] C. Stegall, Duals of certain spaces with the Dunford-Pettis property, Notices Amer. Math. Soc. 19 (1972), A-799.

[26] Talagrand, M., La propriété de Dunford-Pettis dans $\mathcal{C}(K, E)$ et $L^{1}(E)$, Israel $J$. Math. 44, no. 4, 317-321 (1983).

E-mail address: aperalta@ugr.es

Departamento de Análisis Matemático, Facultad de Ciencias, Universidad De Granada, 18071 Granada, Spain.

E-mail address: ignaciov@mat.ucm.es

Departamento de Análisis Matemático, Facultad de Matemáticas, UniverSidad Complutense de Madrid, Madrid 28040, Spain 